\title{
Acute-on-chronic subdural hematoma by spinal anesthesia in a patient with undiagnosed chronic subdural hematoma -A case report-
}

\author{
Il Bong Park, Soo Yeong Moon, Yu Yil Kim, Young Eun Kwon, and Jun Hak Lee \\ Department of Anesthesiology and Pain Medicine, Presbyterian Medical Center, Jeonju, Korea
}

Subdural hematoma is a serious but rare complication of spinal anesthesia. A 70-year-old woman patient underwent elective total knee replacement under spinal anesthesia. At 4 days postoperatively, the patient complained of headache and vomiting. Brain computed tomography revealed an acute-on-chronic subdural hematoma with midline shift. The patient recovered completely after surgical decompression. We report a patient with an undiagnosed chronic subdural hematoma, who developed acute-on-chronic subdural hematoma after spinal anesthesia. (Korean J Anesthesiol 2011; 61: 75-78)

Key Words: Acute-on-chronic, Complication, Spinal anesthesia, Subdural hematoma.

Spinal anesthesia is the most frequently used regional anesthesia that obtunds the stress response, reduces intraoperative blood loss, and decreases the incidence of postoperative thromboembolic events. Since it can reduce the morbidity and mortality of high risk patients, regional anesthesia is often done in pregnant women and old patients [1].

However, spinal anesthesia may cause complications such as back pain, post-dural puncture headache (PDPH), infection, hematoma, nerve injury, and intracranial hemorrhage [1]. Particularly, intracranial hemorrhage, which can occur due to spinal anesthesia, has been reported as a serious, life-threatening complication although its incidence is rare [2-5]. We report the case of a patient who experienced rebleeding from an unexpected chronic subdural hemorrhage that occurred after spinal anesthesia for a left total knee replacement arthroplasty (TKR).

\section{Case Report}

A 70 year-old female patient visited the hospital through the emergency room for acute subdural hematoma. The patient had undergone left TKR under spinal anesthesia for degenerative arthritis in another orthopedic hospital, but symptoms such as headache, vomiting, and decreased consciousness appeared on the fourth day postoperatively. Computed tomography (CT) revealed acute subdural hematoma, and therefore, the patient was transported to our hospital for an emergency operation. According to her past history, she had been on medication

Received: December 14, 2010. Revised: 1st, January 10, 2011; 2nd, January 21, 2011. Accepted: January $24,2011$.

Corresponding author: Yu Yil Kim, M.D., Department of Anesthesiology and Pain Medicine, Presbyterian Medical Center, 300, Junghwasandong 1-ga, Wansan-gu, Jeonju 560-750, Korea. Tel: 82-63-230-8159, Fax: 82-63-230-8169, E-mail: zion_yuyil@yahoo.co.kr

(c) This is an open-access article distributed under the terms of the Creative Commons Attribution Non-Commercial License (http:// creativecommons.org/licenses/by-nc/3.0/), which permits unrestricted non-commercial use, distribution, and reproduction in any medium, provided the original work is properly cited. 
for hypertension that included $100 \mathrm{mg}$ of aspirin from one year before the initial visit and did not show any head trauma or particular clinical or family history. The administration of aspirin stopped seven days before the operation. The complete blood cell count (CBC) and coagulation tests done preoperatively for the left TKR were normal. According to the anesthesiologist from the previous hospital, the spinal anesthesia was done between L4 and L5 in the left lateral decubitus position using a 24-G Quincke needle using the median approach, and the first trial was successful. After verifying the cerebrospinal fluid (CSF) leakage, $12 \mathrm{mg}$ of $0.5 \%$ hyperbaric bupivacaine was injected. There was no pain or abnormal findings during the injection of the drug. Following the spinal anesthesia, the sensory block to the T8 dermatome was verified and then the operation was done. The left TKR took two hours, and the intraoperative vital signs were relatively stable. The estimated blood loss was about $300 \mathrm{ml}$, and two units of packed red blood cell were transfused in the operation room and ward. There were no abnormal findings and the vital signs were stable during the patient's bed rest for the first three days postoperatively. On the fourth day postoperatively, the patient suddenly complained of non-positional headache and vomiting, followed by decreased consciousness. Thus, she had a brain CT, which revealed acute subdural hemorrhage. Then, she was transported to our hospital for an emergency operation. When the patient arrived at our hospital, she was drowsy (or drowsy mentality). A second brain CT revealed a cerebral edema and left deviation from an acute subdural hemorrhage at the right fronto-temporo-parietal lobe. Therefore, an emergency craniectomy and hematoma removal was done (Fig. 1). The operative findings showed a new hemorrhage under the hematoma surrounded by a capsule, that is, an acuteon-chronic subdural hematoma due to the rebleeding. After removing the hematoma, cortical arterial bleeding was found in two regions and hemostasis was done. The patient recovered without any postoperative, neurological sequelae, and she was transferred to another hospital for rehabilitative therapy of the left knee.

\section{Discussion}

Spinal anesthesia is the general procedure that has been used the most frequently since its clinical inception. It provides advantages such as the risk of endotracheal intubation can be avoided and any abnormal signs are quickly recognized because the patient is awake intraoperatively. In particular, spinal anesthesia is the anesthetic method that is preferred in emergency cases when the general anesthesia cannot be done or in case when the risk of general anesthesia is too high. However, spinal anesthesia may also cause various complications from PDPH to intracranial hemorrhage. Among the various complications, intracranial hemorrhage is a serious complication of spinal anesthesia that can lead to death although its incidence is rare. In Korea, there have been reports of subdural hemorrhage [4] and intracerebral hemorrhage [5] due to spinal anesthesia, but there has been no report regarding a case, in which the previous chronic subdural hemorrhage was exacerbated because spinal anesthesia, as in our care report.

Subdural hemorrhage occurs mostly due to the rupture of the bridging dural vein, dural venous sinus wall, or small cerebral cortical vein caused by head trauma. Particularly, intracranial hemorrhage may occur due to some mild, unrecognized trauma usually in cases of the elderly, in which the bridging dural vein tension has increased as the intracranial space widens by physiological brain atrophy. In addition to trauma, subdural hemorrhage can be caused by coagulation disorders, the administration of an anticoagulant drug, or organic lesions such as a tumor, a cerebral aneurysm, and an arterio-

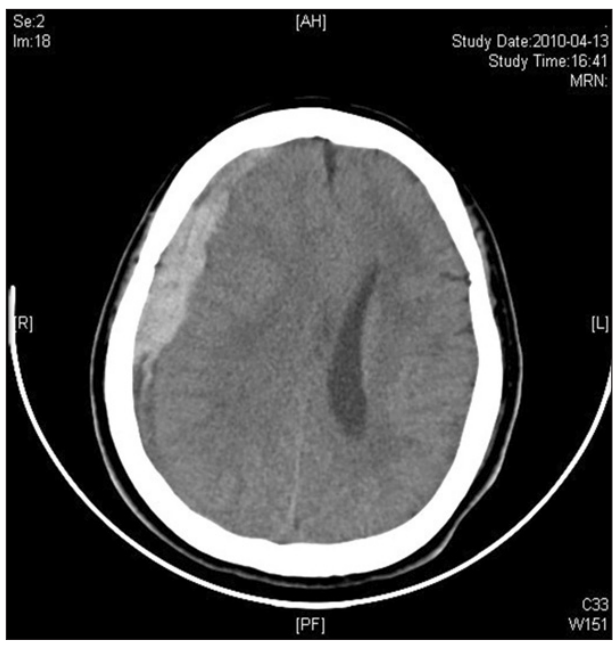

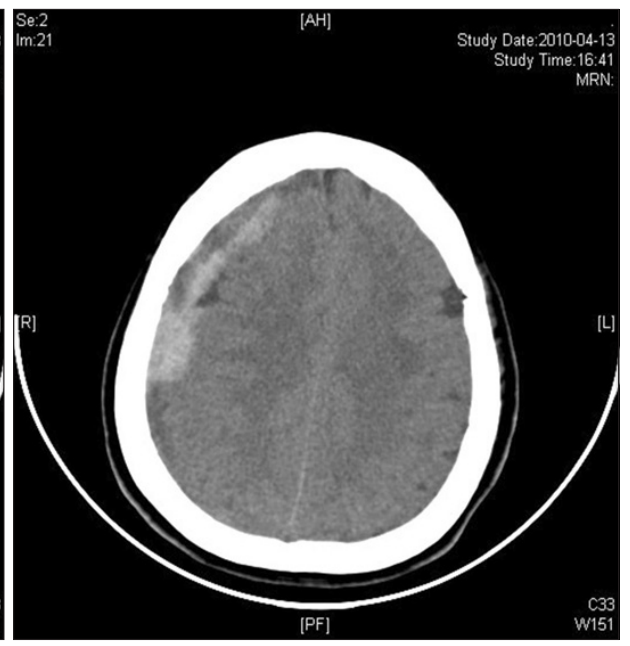

Fig. 1. Computed tomography brain scan shows an acute-on-chronic subdural hematoma with a heterogeneous density. 
venous malformation. Additionally, various factors including decreased intracranial pressure, cerebral atrophy, cranial bone deformation, and CSF leakage may cause or exacerbate the hemorrhage [6]. In the case of our patient, there was no head trauma remembered by the patient and the caregiver. However, since the patient had risk factors such as being over the age of 70 , hypertension, and medicating with aspirins, the subdural hemorrhage might have occurred spontaneously or might have been caused by mild trauma that was not remembered.

In general, subdural hematoma is classified by the lapse of time from the initial head trauma to when symptoms first appear as either an acute subdural hematoma if it occurs within $48-72$ hours, or a subacute hematoma if it occurs 3 to 20 days after the initial trauma, or a chronic hematoma if it occurs three or more weeks after the initial trauma. The time interval until the first symptom appears increases as the age of the patient increases because the hematoma accumulates until the symptoms are clearly identified due to cerebral atrophy. Once a subdural hematoma takes place, a capsule surrounding the hematoma is formed within three weeks, and new blood vessels are generated. During this time, the hematoma is hemolyzed, and the hemolyzed hematoma increases its own volume gradually or disappears by absorption. The hematoma appears in a brain CT as a high-density shadow within one week after its generation, as an isodensity shadow in the second or third week, and as a low-density shadow after the third week but appears as a mixed-density shadow if there is re-bleeding [6]. In our case, a new, acute hematoma was found under the capsule-formed hematoma. Since it takes a considerable time for a capsule of a subdural hematoma to form, the hematoma in our patient could have been formed preoperatively, and a progressing subacute or chronic subdural hematoma could have existed without any symptoms. It is highly probable that the subdural hemorrhage was found in the exacerbated form after general anesthesia or spinal anesthesia because there was a time interval between the head trauma and the appearance of the clinical manifestation. Moreover, a subdural hemorrhage is difficult to find by preoperative tests if there is no clear indication of clinical symptoms in cases of chronic subdural hematomas caused by some unknown trauma or spontaneous hemorrhage, especially in the elderly.

The pathophysiological mechanism of PDPH is not clear, but it may be because of decreased intracranial pressure from CSF leakage. Decreased pressure due to loss of CSF may cause severe traction of the intracranial structure to the caudal side or compensatory expansion of the bridging dural vein and dural venous sinus [7]. Actually, in a patient with decreased intracranial pressure, the CT or MRI revealed a decreased size of the subarachnoidal cistern and cerebral ventricle, an expansion of the dural veins, and a cerebellar amygdalae herniation [8]. Congestion of the bridging vein is caused by a decrease intracranial pressure, which leads to increased intravascular tension and increases the risk of vascular rupture by movement of the brain to the caudal side. In addition, the subdural bridging vein can rupture at the weakest part in the subdural space because the bridging vein in the subdural space is weaker than the bridging vein in the subarachnoid space, when observed under an electronic microscope [9]. However, in our patient, re-bleeding occurred by the rupture of the cerebral cortical artery. The rupture of the cerebral cortical artery might have been caused as the adhesion between the hematoma capsule and the cerebral cortex was separated. Head trauma or whiplash injury may be considered as a cause, but the probability seems low considering that the patient remained in bed rest state after the knee surgery. Another possible cause is the traction of the intracranial structure to the caudal side by the decreased intracranial pressure due to CSF leakage following spinal anesthesia, and it is highly probable that the cerebral cortical artery might have been damaged as the adherence between the hematoma and the cerebral cortex was separated since the hematoma was tightly combined with the dura mater by means of the capsule. We also assume that it might have served as a risk factor in that the chronic subdural hematoma increased the intracranial pressure, and thus, CSF leakage can occur through the dural puncture region following spinal anesthesia.

Moreover, since traction of the pain-sensitive structure or the compensatory expansion of the dural vein by CSF loss are considered as the developing mechanisms for PDPH [10] and the progression from a PDPH to a subdural hematoma was reported [11], a differential diagnosis of a subdural hematoma is necessary if there is a headache or vomiting after spinal anesthesia. PDPH mostly occurs within one or two days after spinal anesthesia, and are aggravated quite typically, when the patient rises from the supine position. In such a case, the headaches improve within 48 hours if the patient placed on bed rest with a sufficient amount of fluid and nonsteroidal analgesics. If necessary, an epidural blood patch can be done. On the contrary, the pattern of headaches for a subdural or intracranial hematoma is independent on the positional change of the patient, and it often accompanies neurological symptoms such as fluctuation in consciousness. In these cases, a brain CT should be done early on to confirm the intracranial hemorrhage. Additionally, an epidural blood patch should only be tried after making a reasonably certain differential diagnosis since it can cause increased intracranial pressure or subdural hemorrhage [12].

Since a diagnostic test is not done in general to check for the possibility of intracranial hemorrhage if there is no distinctive head trauma or neurological symptoms such as decreased 
consciousness preoperatively, intracranial lesions are not found through general preoperative tests. If an undiagnosed subdural hematoma is accompanied as in our case, an intracranial lesion may be exacerbated acutely after the operation, and therefore, the life of the patient could be threatened since the awakening of a patient is retarded after general anesthesia, or the condition of the patient grows rapidly worse after spinal anesthesia. Accurate judgment of the patient's condition preoperatively is important to decrease this risk. In the case of a chronic subdural hematoma, the initial symptoms generally include continuous headaches, vomiting, mild hemiplegia, papilledema, and language disorder. However, the elderly can show different symptoms such as confusion, memory disorder, decreased attention, and changed personality. This may lead to a misdiagnosis as the foregoing symptoms are similar to those of dementia or other psychotic disorders [6]. Hence, in amnesic disorders, when decreased attention and changed personality are observed, it may be necessary to perform diagnostic testing preoperatively.

In our patient, the operative finding was re-bleeding under the existing hematoma capsule. It is highly probable that the re-bleeding might have occurred as the previous hematoma was separated from the cerebral cortex due to the loss of CSF after the spinal anesthesia since no head trauma was found postoperatively and the blood coagulation test revealed normal findings.

In conclusion, an anesthesiologist should keep in mind that there can be an asymptomatic intracranial hemorrhage in a patient and that an intracranial lesion may be exacerbated or newly occurred even during spinal anesthesia. Even when spinal anesthesia is done, a preoperative evaluation should be thoroughly done. Moreover, the perioperative conditions of the patient should be carefully monitored and evaluated, and then rapid and appropriate treatment should be done early if an abnormal situation occurs. Particularly in elderly patients, recent changes in cognitive functions of the brain and general conditions should not be neglected. Moreover, progression should be actively monitored when a post-puncture headache occurs, and an additional test should be done early on to differentiate the headache from other causes if the headache continues or its pattern changes.

\section{References}

1. Bernards CM. Epidural and spinal anesthesia. In: Clinical Anesthesia. 6th ed. Edited by Barash PG, Cullen BF, Stoelting RK: Philadelphia, Lippincott Williams \& Wilkins. 2009, pp 927-54.

2. Zeidan A, Chaaban M, Farhat O, Baraka A. Cerebral rebleeding by spinal anesthesia in a patient with undiagnosed chronic subdural hematoma. Anesthesiology 2006; 104: 613-4.

3. Tan ST, Hung CT. Acute-on-chronic subdural haematoma: a rare complication after spinal anaesthesia. Hong Kong Med J 2003; 9: 384-6.

4. Kwak SW, Suh MK, Kim SS. Cranial subdural hematoma developing after spinal anesthesia. Korean J Anesthesiol 2009; 56: 92-5.

5. Kim EJ, Chang IY. Intracerebral hemorrhage after vesicolitholapaxy under spinal anesthesia. Korean J Anesthesiol 2006; 51: 379-82.

6. The Korean Neurosurgical Society. Neurosurgery. 3rd ed. Seoul, Jungang Moonhwa Co. 2005, pp 435-9.

7. Lay CL, Campbell JK, Mokri B. Low cerebrospinal fluid pressure headache. In: Headache. 2nd ed. Edited by Goadsby PJ, Silberstein SD: Boston, Butterworth-Heinemann. 1997, pp 355-67.

8. Reina MA, Alvarez-Linera J, López A, Benito-León J, De Andrés JA, Sola RG. Magnetic resonance in dural post-puncture headache in patient with cerebrospinal fluid hypotension. Rev Esp Anestesiol Reanim 2002; 49: 89-100.

9. Yamashima T, Friede RL. Why do bridging veins rupture into the virtual subdural space? J Neurol Neurosurg Psychiatry 1984; 47: 121-7.

10. Turnbull DK, Shepherd DB. Post-dural puncture headache: pathogenesis, prevention and treatment. Br J Anaesth 2003; 91: 71829.

11. Zeidan A, Farhat O, Maaliki H, Baraka A. Does postdural puncture headache left untreated lead to subdural hematoma? Case report and review of the literature. Int J Obstet Anesth 2006; 15: 50-8.

12. Kim YS, Han KR, Kim C. A case of subdural hematoma after epidural blood patch in a spontaneous intracranial hypotensive patient. Korean J Pain 2007; 20: 235-9. 